\title{
RESPONSE OF PLATES UNDER STATISTICALLY UNSYNCHRONIZED UNIFORM RANDOM LOADS USING MONTE-CARLO SIMULATION
}

\author{
JeAn-Michel Dhainaut \\ Mechanical Engineering Department, Embry-Riddle University \\ Daytona Beach, FL 32114, USA \\ dhain460@erau.edu \\ Guangfeng Cheng \\ Jefferson Lab \\ Newport News, VA 23606, USA \\ cheng@jlab.org \\ Chun MeI \\ Old Dominion University \\ Norfolk, VA 52329, USA \\ cmei@odu.edu
}

[Received: February 8, 2007]

\begin{abstract}
Classic solutions of the responses of plates subjected to uniform random pressure are generally confined to simple boundary conditions where the assumed mode shape solutions are known. Even in those simple cases, response solutions have neglected the asymmetric modes for rectangular plates with symmetric boundary conditions due to mathematical limitations. To the best know;edge of the authors, the present paper presents for the first time the random response and fatigue life prediction for plates subjected to unsynchronized and statistically uncorrelated uniform random loads. A linear finite element formulation combined with the Monte Carlo simulation is employed to determine the stress response, and the stochastic Palmgrem-Miner reliability model is used for the fatigue analysis. A simply supported rectangular plate is studied first in detail to show the contributions of both symmetric and asymmetric modes due to the unsynchronized loading. A clampedsimply supported plate is analyzed next to show the versatility of the present approach. In both cases, a significant reduction on the responses and increase in fatigue life was observed as compared to corresponding results from the uniform synchronized loading condition. Results also include RMS displacement and stress values, power spectral densities, probability density functions, and rainflow plots. Computational burden due to the unsynchronized random pressure fluctuations required the use of parallel computing capabilities.
\end{abstract}

Mathematical Subject Classification: 05C38, 15A15

Keywords: unsynchronized, random, fatigue life

\section{INTRODUCTION}

Currently the random acoustic pressure for sonic fatigue design and analysis of beam, plate [1, 2, 3, 4, 5, 6, 7] and shallow shell [8] structures (linear or non-linear) is often considered uniformly distributed over the surface of the structure and synchronized in 
time. In addition, the random loading is generally assumed as a truncated Gaussian white noise. In two previous experiments, two clamped square aluminum plates were tested in the Wideband Noise Test Chamber of the Flight Dynamics Laboratory Sonic Fatigue Facility at WPAFB. The mean-square strains versus non-dimensional Sound Pressure Level (SPL) showed good agreement among the PDE/Galerkin/Equivalent Linearization (EL) analytical results and the experimental data [2, 9. A steel beam rigidly clamped in a clamping fixture and mounted on a vibration shaker was also recently tested. Excellent agreement of the strain Power Spectral Density (PSD) was obtained among the experimental data and the two finite element modal results [3, 10]. In both experiments, the loading could be considered as synchronized in time and uniformly distributed, thus the asymmetric natural bending modes of the panel were not considered in the analysis. By synchronized load it is meant that the simulated random pressure load is generated from one seed number [1, 3, 7, 8, 10] (see Section III. C) for the whole surface area of the panel, and at each time step the pressure load on the beam, plate or shell is uniform. On the other hand, let us use the long period of steady rain falling on top of a flat small roof as an illustration of the unsynchronized uniform random load. The pressure of the raindrops falling on the roof surface is certainly random in nature, with more or less a fairly uniform pressure distribution in the random sense (white noise, but not identical time history). It cannot be simulated as a random uniform pressure synchronized in time coming from only one seed number, but rather as a random load with different seed number for each raindrop in an ideal simulation case. Another example of uniform random load unsynchronized in time is the 30,000 to 40,000 marathon runners across a long-span bridge, especially cablesupported. For those cases, the asymmetric as well as the symmetric modes are excited and they should be considered in the analysis. For panel design in practice, the highest measured or estimated PSD level should be used, and it may also be reasonable to assume that the PSD is a band-limited white noise, since the contribution from the high frequency modes to the panel response is usually small. Influences of acoustic pressure with nonwhite PSD (synchronized) on maximum deflection, stress/strain and fatigue life have been investigated recently for plates[?] and shallow shells.[?]. This paper studies the random response and fatigue life of isotropic rectangular plates subjected to a uniformly distributed random pressure load unsynchronized in time. Results show that the Root Mean Square (RMS) deflections and maximum stress are reduced and the fatigue life is increased for the unsynchronized as compared with the traditional synchronized loading case.

\section{Analytical solution}

The mathematical difficulties in considering the asymmetric modes in the response of a plate under uniformly distributed random load (white-noise) using the classic analytical method is briefly explained first. Considering the linear vibrations of a SimplySupported (S-S) isotropic plate subjected to a uniformly distributed random pressure $P(t)$. The transverse displacement $w(x, y, t)$ of a $a \times b \times h$ (length $\times$ width $\times$ thickness $)$ 
panel can be represented as

$$
w(x, y, t)=\sum_{r=1}^{m} \sum_{s=1}^{n} q_{r, s}(t) \sin \left(\frac{r \pi x}{a}\right) \sin \left(\frac{s \pi y}{b}\right),(r, s)=1,2,3 \ldots
$$

The classic analytical partial differential equation (PDE) and normal mode approach leads to the modal equations for the damped motion of the panel as

$$
\ddot{q}_{r, s}+2 \zeta_{r, s} \omega_{r, s} \dot{q}_{r, s}+\omega_{r, s}^{2} q_{r, s}=\frac{\gamma_{r, s}}{m_{r, s}} P(t), \quad(r, s)=1,3,5 \ldots
$$

By setting $(r, s)=m$ and $(k, l)=n$, the mean-square values of the modal amplitudes are

$$
E\left[q_{m}^{2}\right]=\frac{\gamma_{m}^{2} S_{0}}{8 m^{2} \zeta_{m} \omega_{m}^{3}}
$$

and

$$
\begin{aligned}
& E\left[q_{m} q_{n}\right]= \\
& \quad=\frac{\gamma_{m} \gamma_{n}\left(\zeta_{m} \omega_{m}+\zeta_{n} \omega_{n}\right) S_{0}}{m_{m} m_{n}\left[\left(\omega_{m}^{2}-\omega_{n}^{2}\right)^{2}+4 \omega_{m} \omega_{n}\left(\zeta_{m} \omega_{m}+\zeta_{n} \omega_{n}\right)\left(\zeta_{m} \omega_{n}+\zeta_{n} \omega_{m}\right)\right]}
\end{aligned}
$$

where $\zeta_{m}$ is the modal damping coefficient, $\omega_{m}$ the natural frequency corresponding to the $(r, s)$ mode, respectively, and $m=\frac{\rho h a b}{4}$ the constant modal mass. The coefficient $\gamma_{r, s}=\gamma_{m}$ is the result from the application of the PDE/normal mode approach as

$$
\int_{0}^{a} \int_{0}^{b} P(t) \sin \left(\frac{r \pi x}{a}\right) \sin \left(\frac{s \pi y}{b}\right) d x d y= \begin{cases}P(t) \gamma_{m} & \text { for }(r, s)=\text { odd } \\ 0 & \text { for } r \text { or } s=\text { even }\end{cases}
$$

where $\gamma_{m}=\frac{4 a b}{r s \pi^{2}}$. The $\gamma_{m}$ value from equations $(3)$ and 5 implies that only the symmetric modes ( $r$ and $s=$ odd) are excited with the uniformly distributed random pressure $P(t)$ synchronized in time since for asymmetric modes, ( $r$ or $s=$ even), the integration of equation (5) over the surface of the panel is zero.

The definition of $P(t)$ is a rather restricted case of random load since at any instant time the pressure is constant at any location of the panel, and thus $P(t)$ is statistically synchronized in time. This synchronized uniform random pressure $P(t)$ does not fit the more general stochastic definition of band-limited white noise pressure,

$$
S_{p}(\xi, \eta, f)= \begin{cases}S_{0} & \text { if } 0 \leqslant f \leqslant f_{u} \\ 0 & \text { if } f<0 \text { or } f>f_{u} \\ \xi=x_{1}-x_{2} & \\ \eta=y_{1}-y_{2} & \end{cases}
$$

where the spatial separations are denoted by $\xi=x_{1-} x_{2}, \gamma=y_{1-} y_{2}$, and the upper cutoff frequency in hertz $(\mathrm{Hz})$ by $f_{u}$. The auto-spectral density function of the random excitation is defined as

$$
S_{0}=P_{0}^{2} 10^{S P L / 10}
$$


where SPL denotes the sound pressure level in decibels $(\mathrm{dB})$, and $P_{0}$ is the reference pressure equal to $2.9008 \times 10^{-9} \mathrm{psi}(20 \mu \mathrm{Pa})$. Explicitly, the time histories of random pressure $P(x, y, t)$ at any two points of the panel could be different and statistically unsynchronized in time, however, their PSD values $\left(S_{o}\right)$ should be the same. This statistically unsynchronized uniformly distributed random pressure would certainly excite the asymmetric ( $r$ or $s=$ even) as well as symmetric ( $r$ or $s=$ odd) modes. However, as it can be easily seen from equation (5) that the asymmetric modes would not be considered in the analytical PDE/normal mode solution. The steady rain and the marathon runners discussed earlier are two examples of statistically unsynchronized uniform random loads just addressed.

\section{Formulation and Simulation}

\subsection{Finite Element Formulation.}

\section{Structural Node Degree of Freedom (DOF)}

The finite element employed in the present study is the $\mathrm{C}^{1}$-conforming Bogner-FoxSchmit 11] (BFS) plate element, which has a total of 16 bending DOF $\left\{w_{b}\right\}$ :

$$
\left\{w_{b}\right\}=\left\{w_{k} w_{x k} w,_{y k} w_{x y k}\right\}^{T}
$$

Applying the principle of virtual work, the system equations of motion are derived,

$$
\left[M_{b}\right]\left\{\ddot{W}_{b}\right\}+\left[K_{b}\right]\left\{W_{b}\right\}=\{P\}
$$

where $\left[M_{b}\right]$ and $\left[K_{b}\right]$ are the system consistent mass and stiffness matrices, and $\{P\}$ is the load vector for uniform random loading.

\section{Modal DOF}

The system equations of motion in the structural DOF are then transformed into a set of truncated modal coordinates by expressing the plate response as a linear combination of some base functions as

$$
\left\{W_{b}\right\}=\sum_{m=1}^{l} q_{m}(t)\left\{\phi_{b}\right\}^{(m)}=[\Phi]\{q\}
$$

where $\left\{\phi_{b}\right\}^{(m)}$ corresponds to the $m$-th normal mode of the linear vibration problem. Adding a damping matrix $2 \zeta_{m} \omega_{m}[I]$ where the modal damping $\zeta_{m}$ can be determined experimentally or from previous experience. The equations of motion, equation (9), are reduced to a set of uncoupled modal equations as

$$
\left[\bar{M}_{b}\right]\{\ddot{q}\}+2 \zeta_{r} \omega_{r}\left[\bar{M}_{b}\right]\{\dot{q}\}+\left[\bar{K}_{b}\right]\{q\}=\{\bar{P}\}
$$

where the diagonal modal mass and stiffness matrices are

$$
\left(\left[\bar{M}_{b}\right],\left[\bar{K}_{b}\right]\right)=[\Phi]^{T}\left(\left[M_{b}\right],\left[K_{b}\right]\right)[\Phi]
$$

and the modal random load vector is

$$
\{\bar{P}\}=[\Phi]^{T}\{P\}
$$

The modal displacement $\{q\}$ in equation (11) is obtained by the fourth order RungeKutta (RK4) numerical integration scheme. The system bending displacement $\left\{W_{b}\right\}$ 
is then calculated with equation $(10)$, and the stress vector $\{\sigma\}$ at the element level is evaluated with

$$
\{M\}=[D]\{\kappa\},\{\sigma\}=\left( \pm \frac{6}{h^{2}}\right)\{M\}
$$

where $h$ is the thickness, $\{M\}$ the bending moment vector, $\{\kappa\}$ the curvature, and $[D]$ the bending stiffness matrix of the plate. For the displacement based finite element method, the stress calculation is not as accurate as displacement calculation. According to Barlow 12 the stresses are more precise if calculated at the Barlow points and then extrapolated to the nodal points or other desired point.

\subsection{Fatigue Life Estimation - RFC Method.}

The Palmgren-Miner 13 cumulative damage theory method is employed for fatigue life analysis in the time domain

$$
D=\sum_{i=1}^{k} \frac{n_{i}}{N_{i}}=1.0
$$

where $D$ is the damage and $n_{i}$ and $N_{i}$ are the actual number of cycles at a given stress level and the number of total cycles at which failure occurs at the same stress level. The stress/strain versus the number of cycles to failure (S-N) curve normally takes the form,

$$
N=K / S^{\beta}
$$

where the material constants $K$ and $\beta$ are determined experimentally. For random response, the Palmgren-Miner [13] theory is rewritten as

$$
E[D(t)]=\frac{1}{K} \int_{0}^{\infty} p(s) s^{\beta} d s=E\left[\frac{1}{K} \sum p(s) s^{\beta}\right]=E\left[\frac{1}{K} \sum s_{k}^{\beta}\right]
$$

where $p(s)$ is the stress/strain range Probability Distribution Function (PDF). Based on equations 15 and 17 the simplest fatigue life estimate is

$$
T^{f}=\frac{1}{E[D(t)]}
$$

Some of the cycles counting methods to predict $p(s)$ are: (i) the peak counting method, (ii) the range counting method, and (iii) the Rainflow Counting Cycles (RFC) method. Langley and McWilliam [14 showed that the first two methods give similar results for narrow-band processes, but quite different for a wide-band process. The RFC method uses a specific counting scheme to account for effective stress ranges and identified stress cycles related to closed hysteresis loops in the stress-strain curves. The validity of the RFC method was studied in detail by Dowling 15 where the accuracy of fatigue life predictors was based on eight commonly used cycle counting methods. Dowling concluded that the RFC method was the only accurate method for wide-band processes. The RFC algorithm used in this paper was provided as part of the Wave Analysis for Fatigue analysis and Oceanography [16] (WAFO) toolbox. In addition, Bishop and Sherratt [17] showed that fatigue life of wide-band Gaussian signal using the RFC yields to the most realistic estimates. 


\subsection{Synchronized Random Pressure Generation.}

Consider a uniformly distributed random pressure $P(t)$ that is characterized by the auto-spectral density function defined by equation (6) and (7). A simple process possessing such a PSD is a truncated white noise and its samples are assumed here to be drawn according to a Gaussian distribution. An algorithm [1, 3] that simulates a random pressure using complex numbers with independent random phase angles uniformly distributed between 0 and $2 \pi$ is used to generate the band-limited truncated white noise [1, 3, 8]. The algorithm uses a random number generator that needs an initial seed number (ISEED). Once the random pressure time history is generated the average value of the auto-spectral density is calculated and compared to $S_{0}$ for a given SPL. Each pressure time history had a time history of 1 second, a cut-off frequency of $4096 \operatorname{Hertz}(\mathrm{Hz})$, a time step $\Delta t=1 / 2^{16}$ second, and a total of 65536 (NPT) pressure data points.

\subsection{Unsynchronized Random Pressure Generation.}

The unsynchronized random load generation follows alike procedure as the synchronized load case, except that for each plate element, a random load is generated with a different ISEED number. Using different ISEED numbers guaranties that each random pressure time history to be different from one another as shown in Figure 1.
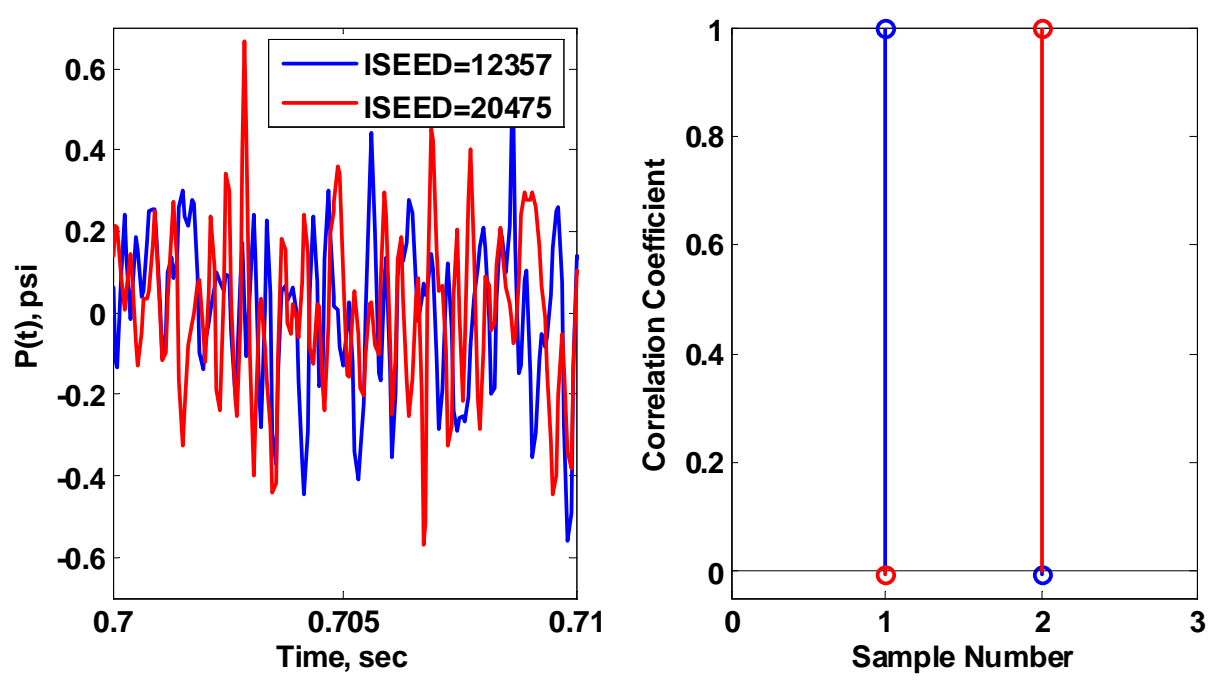

Figure 1. Random load generation for ISEED $=14407$ and ISEED $=12357$

This phenomenon was referred in the introduction as the simulation of the raindrops and/or the marathon runners' steps on a cable-supported bridge. Table 1 shows that pressure histories from 5 different ISEED numbers have a PSD equal to mean value of $S_{0}$ at $\mathrm{SPL}=100 \mathrm{~dB}$. 
Table 1. Load generation from 5 different ISEED numbers

\begin{tabular}{|c|c|c|c|c|c|}
\hline ISSED & Analytical & $\boldsymbol{P S D}^{*}$ & Time history $^{* *}$ & ${ }^{*} \boldsymbol{E r} \boldsymbol{r}$. \% & ${ }^{* *}$ Err. \% \\
\hline $\mathbf{2 0 3 9 2}$ & $3.44651799 \mathrm{e}-2$ & $3.446530047 \mathrm{e}-2$ & $3.453362 \mathrm{e}-2$ & 0.00034 & -0.1985 \\
\hline $\mathbf{2 0 4 6 2}$ & $3.44651799 \mathrm{e}-2$ & $3.446530048 \mathrm{e}-2$ & $3.459602 \mathrm{e}-2$ & 0.00034 & -0.3796 \\
\hline $\mathbf{2 0 5 0 6}$ & $3.44651799 \mathrm{e}-2$ & $3.446530047 \mathrm{e}-2$ & $3.435795 \mathrm{e}-2$ & 0.00034 & 0.3111 \\
\hline $\mathbf{2 0 5 2 7}$ & $3.44651799 \mathrm{e}-2$ & $3.446530047 \mathrm{e}-2$ & $3.445678 \mathrm{e}-2$ & 0.00034 & 0.02435 \\
\hline $\mathbf{1 2 3 5 7}$ & $3.44651799 \mathrm{e}-2$ & $3.44653004 \mathrm{e}-2$ & $3.446085 \mathrm{e}-2$ & 0.00034 & 0.01254 \\
\hline
\end{tabular}

Analytical: Power $=\left(\mathrm{P}_{r e f}^{2} 10^{S P L / 10}\right) \times f_{u}, \mathrm{PSD}^{*}:$ Power $=\operatorname{mean}\left(\mathrm{S}_{0}\right) \times f_{u}$, and Time history $^{* *}:$ Power $=\mathrm{P}(\mathrm{t})^{2} / \mathrm{NPT}$

A more detailed analysis using the correlation coefficient command in MATLAB has shown that the pressure time histories were statistically uncorrelated to each other as shown in Figure 1. For instance, if a generated pressure time history was compared with fifty others (all with different ISEED numbers), the lowest correlation coefficient found was 0.0011 and the highest 0.0264 , where a correlation coefficient of 0 indicates no relationship and a coefficient of 1.0 indicates the highest possible relationship (correlation factor of the pressure time histories with each other).

\subsection{Parallel Computations.}

The computational burden caused by the storage of the random loads from different ISEED numbers was resolved by the use of parallel computing facilities at Embry-Riddle Aeronautical University. In Matlab the Distributed Computing Toolbox (DCT) and the Distributed Computing Engine (DCE) enable to develop parallel MATLAB applications and execute them on a cluster of computers without leaving the Matlab environment. A local UNIX machine was used to connect to the DCE installed in the cluster. The MCS-FE code was run into two workers (nodes) $4 \mathrm{~GB}$ Ram memory each; the first worker was used as storage for the huge amount of data points contained in the load matrix. (There are 1024 columns each containing 16384 points). The second worker is used exclusively to run the modal finite element numerical simulation. The program is at the first stage to be adapted for parallel computing capabilities. At this stage the program was modified such that at each time step the FE-MCS was able to call the corresponding random pressure values contained in the load matrix. In a near future the program would be optimized to be able to run on multiples nodes which will significantly increase the number of degree of freedom (or mesh size) and it will decrease the computational time.

\section{Results And Discussion}

The responses of a plate under the synchronized uniform random pressure are studied first. Results are compared between the analytical solution (see section II) and the Finite Element code (FE) which uses the Monte Carlo Simulation (MCS). Next, the same panel is subject to an unsynchronized random load, and results are quantitatively compared to previous ones. Finally, the FE modal formulation is generalized by the study of the response of a panel with mixed (asymmetric) boundary conditions under the unsynchronized uniform random pressure. 
4.1. Validation. A Simply-Supported (S-S) plate was first selected for validation because some preliminary results can be compared with the analytical solution. The aluminum plate has dimension 15 in. $\times 12$ in. $\times 0.040$ in. $(38.1 \mathrm{~cm} \times 30.48 \mathrm{~cm} \times$ $0.1016 \mathrm{~cm})$. The material properties are Young's modulus $E=10.587 \times 10^{6} \mathrm{psi}(73$ GPa), density $\rho=2.588 \times 10^{-4} \mathrm{lbf}-\mathrm{sec}^{2} /$ in. $^{4}\left(2763 \mathrm{~kg} / \mathrm{m}^{3}\right)$, Poisson ratio $\nu=0.30$. A constant modal damping ratio of $\zeta_{r}=0.02$ is applied. The panel is modeled with a convenient $32 \times 32$ mesh (1024 BFS elements). The lowest twenty-two natural frequencies and corresponding modes are given in Table 2.

Table 2. Lowest twenty-two S-S panel modes and frequencies in $\mathrm{Hz}(32 \times 32$ mesh)

\begin{tabular}{|c|c|c|c|c|c|c|c|c|c|c|c|}
\hline Mode & $\mathbf{( 1 , 1 )}$ & $\mathbf{( 2 , 1 )}$ & $\mathbf{( 1 , 2 )}$ & $\mathbf{( 2 , 2 )}$ & $\mathbf{( 3 , 1 )}$ & $\mathbf{( 1 , 3 )}$ & $\mathbf{( 3 , 2 )}$ & $\mathbf{( 4 , 1 )}$ & $\mathbf{( 2 , 3})$ & $\mathbf{( 4 , 2 )}$ & $\mathbf{( 3 , 3 )}$ \\
\hline FEM & 43.8 & 95.07 & 123.9 & 175.2 & 180.5 & 257.5 & 260.7 & 300.2 & 308.7 & 380.4 & 394.2 \\
\hline $\begin{array}{c}\text { Analy- } \\
\text { tical }\end{array}$ & 43.8 & 95.1 & 123.9 & 175.2 & 180.5 & 257.4 & 260.7 & 300.2 & 308.7 & 380.3 & 394.2 \\
\hline Mode & $\mathbf{( 1 , 4 )}$ & $\mathbf{( 5 , 1 )}$ & $\mathbf{( 2 , 4 )}$ & $\mathbf{( 4 , 3 )}$ & $\mathbf{( 5 , 2 )}$ & $\mathbf{( 3 , 4 )}$ & $\mathbf{( 6 , 1 )}$ & $\mathbf{( 5 , 3 )}$ & $\mathbf{( 1 , 5 )}$ & $\mathbf{( 4 , 4 )}$ & $\mathbf{( 6 , 2 )}$ \\
\hline FEM & 444.4 & 454.0 & 495.7 & 513.8 & 534.1 & 581.1 & 642.1 & 667.7 & 684.8 & 700.8 & 722.2 \\
\hline $\begin{array}{c}\text { Analy- } \\
\text { tical }\end{array}$ & 444.4 & 454.0 & 495.7 & 513.8 & 534.1 & 581.1 & 642.0 & 667.7 & 684.7 & 700.8 & 722.1 \\
\hline
\end{tabular}

The number of modal coordinates to be included in the analysis for converged deflection and stress is studied first. The RMS maximum non-dimensional deflection $\left(\mathrm{W}_{\max } / \mathrm{h}\right)$ and the RMS maximum stress $\left(\sigma_{y}\right.$ at the plate center) for different number of modes under a synchronized random load of intensity SPL $=100 \mathrm{~dB}$ are given in Table 3. Analytical solutions (section II) for 1, 2, 4 and 5 symmetric modes are also tabulated for validation of the present modal Finite Element Model (FEM).

Table 3. Verification and modal convergence for S-S panel under synchronized loading $(32 \times 32$ mesh $)$

\begin{tabular}{|c|c|c|c|c|}
\hline $\begin{array}{c}\mathrm{SPL}=100 \mathrm{~dB} \\
\text { Modes }\end{array}$ & $\begin{array}{c}\text { Analytical } \\
\text { RMS } \\
\left(\mathbf{W}_{\max } / \mathbf{h}\right)\end{array}$ & $\begin{array}{c}\text { Analytical } \\
\text { RMS(Stress) } \\
\text { psi }\end{array}$ & $\begin{array}{c}\text { FEM } \\
\text { RMS } \\
\left(\mathbf{W}_{\max } / \mathbf{h}\right)\end{array}$ & $\begin{array}{c}\text { FEM } \\
\text { RMS(Stress) } \\
\text { psi }\end{array}$ \\
\hline$(1,1)$ & 0.6219 & $473.105(3.26 \mathrm{MPa})$ & 0.6220 & $474.472(3.27 \mathrm{MPa})$ \\
\hline$(1,1),(3,1)$ & 0.6227 & $475.043(3.28 \mathrm{MPa})$ & 0.6227 & $476.690(3.28 \mathrm{MPa})$ \\
\hline $\begin{array}{l}(1,1),(3,1), \\
(1,3),(3,3)\end{array}$ & 0.6228 & $483.010(3.33 \mathrm{MPa})$ & 0.6229 & $485.220(3.35 \mathrm{MPa})$ \\
\hline $\begin{array}{c}(1,1),(3,1) \\
(1,3),(3,3),(5,1)\end{array}$ & 0.6228 & $483.070(3.33 \mathrm{MPa})$ & 0.6230 & $484.950(3.34 \mathrm{MPa})$ \\
\hline Lowest 4 modes & 0.6219 & $473.105(3.26 \mathrm{MPa})$ & 0.6220 & $474.472(3.27 \mathrm{MPa})$ \\
\hline Lowest 5 modes & 0.6227 & $475.090(3.28 \mathrm{MPa})$ & 0.6227 & $476.690(3.28 \mathrm{MPa})$ \\
\hline Lowest 11 modes & 0.6228 & $483.010(3.33 \mathrm{MPa})$ & 0.6229 & $485.220(3.35 \mathrm{MPa})$ \\
\hline Lowest 13 modes & 0.6228 & $483.070(3.33 \mathrm{MPa})$ & 0.6230 & $484.950(3.34 \mathrm{MPa})$ \\
\hline
\end{tabular}

Results show that the lowest four symmetric modes $(1,1),(3,1),(1,3)$, and $(3,3)$ are sufficient for a converged displacement and stress solutions, and that the asymmetric modes $(2,1),(1,2),(2,2),(3,2),(4,1),(2,3),(4,2),(1,4)$ and $(5,1)$ have no appreciable contribution to the maximum displacement response. The modal convergence based on stress also indicated that four symmetric modes are sufficient for converging stress response. Since the present paper deals with fatigue life predictions, which are based 
on stress ranges, equation (17), four symmetric modes are considered for converged stress calculations. In the RMS calculations, the transient part of the responses has been omitted by dropping the first 8192 data points or 0.125 second (sec). This means that the total useful time of data is $0.875 \mathrm{sec}$ for each sample. This same procedure is adopted for all subsequent results in the paper. Fatigue life prediction results are obtained by evaluating the mean of the RMS values of ten samples.

Two other considerations for accurate and converged response predictions were also addressed: the number of elements and the integration time step. It is found that a discretization of the panel into 1024 BFS elements is more than adequate since 384 elements ( $24 \times 16$ mesh) should be sufficient for converged responses. The selection of the time step has observed two criteria: 1) the Nyquist-Shannon sampling theorem, which basically states that it is necessary to sample a time sequence at least two times faster than the highest frequency present in the modal expansion $(394.18 \mathrm{~Hz})$, and 2) two consecutive time steps $\left(\Delta t_{2}=\Delta t_{1} / 2\right)$, for a same pressure load, should give identical time history responses. It was found that a time step of $\Delta t=1 / 2^{16}=$ $1.52 \times 10^{-5}$ sec satisfies the above two requirements.

\subsection{Unsynchronized Response of Simply Supported Panel.}

The same panel $(32 \times 32$ mesh $)$ was subjected to an unsynchronized sample random pressure load by the procedure described in section III.D. Results in this section are obtained from a unique random load file containing 1024 uncorrelated random pressure loads for the $32 \times 32=1024$ elements. This means that RMS values are always calculated from the same random load file (always same ISEED numbers), and can consequently be used for modal and mesh convergence purposes. However, since the RMS values are based on only one load sample their accuracy (RMS values) cannot be considered as accurate. As it will be explained in a later section, the accuracy is improved by applying the MCS technique by taking many samples. Table 4 shows the RMS values of the maximum stress computed from different mesh sizes. It is observed that a stress mesh convergence is not obtained by a mesh refinement of $32 \times 32$, which is the maximum mesh size that a Dell Precision 450 (Intel Xeon processor $533 \mathrm{MHz}$ ) with $4 \mathrm{~GB}$ of ram memory could model. This limitation could be

Table 4. Mesh and modal convergence for stress of S-S panel under unsynchronized loading

\begin{tabular}{|c|c|c|c|}
\hline Mesh Size & $\begin{array}{c}16 \times 16 \text { mesh } \\
\text { RMS }(\text { Stress }), \text { psi }\end{array}$ & $\begin{array}{c}24 \times 20 \text { mesh } \\
\text { RMS(Stress) }, \text { psi }\end{array}$ & $\begin{array}{c}32 \times 32 \text { mesh } \\
\text { RMS(Stress), psi }\end{array}$ \\
\hline Lowest 11 modes & $84.34(0.582 \mathrm{MPa})$ & $62.65(0.432 \mathrm{MPa})$ & $17.62(0.121 \mathrm{MPa})$ \\
\hline Lowest 18 modes & $84.77(0.585 \mathrm{MPa})$ & $62.82(0.433 \mathrm{MPa})$ & $17.78(0.123 \mathrm{MPa})$ \\
\hline Lowest 20 modes & $87.97(0.607 \mathrm{MPa})$ & $64.37(0.444 \mathrm{MPa})$ & $19.14(0.132 \mathrm{MPa})$ \\
\hline Lowest 22 modes & $87.97(0.607 \mathrm{MPa})$ & $64.37(0.444 \mathrm{MPa})$ & 19.14 (0.132 MPa) \\
\hline
\end{tabular}

overcome by the use of Parallel Computing Methods (PCM), but it will not be addressed in this paper. The main goal of the present work is to show the reduction in RMS responses and increase in fatigue life prediction due to the unsynchronized characteristics of the random load. However, it was observed that 20 modes were sufficient for modal convergence. Consequently, unsynchronized random results will 
be limited to a $32 \times 32$ mesh size with 20 modes, where the highest two modes are $(5,3)$ with a frequency of $667.65 \mathrm{~Hz}$, and $(1,5)$ with frequency $684.74 \mathrm{~Hz}$, respectively. These two modes indicate that the $32 \times 32$ mesh scheme is adequate for numerical simulation.

In order to show the contribution from asymmetric modes other than the lowest four symmetric modes $(1,1),(3,1),(1,3)$, and $(3,3)$, the deflection and stress PSD plots at $x=15 / 4$ in. and $y=12 / 4$ in., and at the center of the panel for the synchronized and unsynchronized loads at $\mathrm{SPL}=100 \mathrm{~dB}$ are shown in Figure 2. The displacement figure at $x=15 / 4 \mathrm{in}$. and $y=12 / 4$ in. $(x=38.1 / 4 \mathrm{~cm} \& y=30.48 / 4$ $\mathrm{cm})$ clearly indicates that the unsynchronized loading case excited the modes $(2,1)$, $(1,2)$, and $(2,3)$ in addition to the lowest five symmetric modes. A close observation reveals that the mode $(4,1)$ is also excited, but it does not appear on the PSD since the analysis is at the node point for mode $(4,1)$. Stress PSD at $x=15 / 4 \mathrm{in}$. and $y=12 / 4$ in. $(x=38.1 / 4 \mathrm{~cm} \mathrm{\&} y=30.48 / 4 \mathrm{~cm})$ shows that at this location the stress is still influenced by the symmetric modes $(3,1)$ for the synchronized loading case, while for the unsynchronized case the contribution from the of symmetric and asymmetric modes, $(2,1),(1,2),(4,1)$, is more or less evenly distributed. Finally, the stress PSD indicates that the area under the curve (Power $\left.=S_{0} \times \Delta f\right)$ is much lower for the unsynchronized than for the synchronized load case. This implies that the fatigue life would then be higher for the unsynchronized loading case [17.
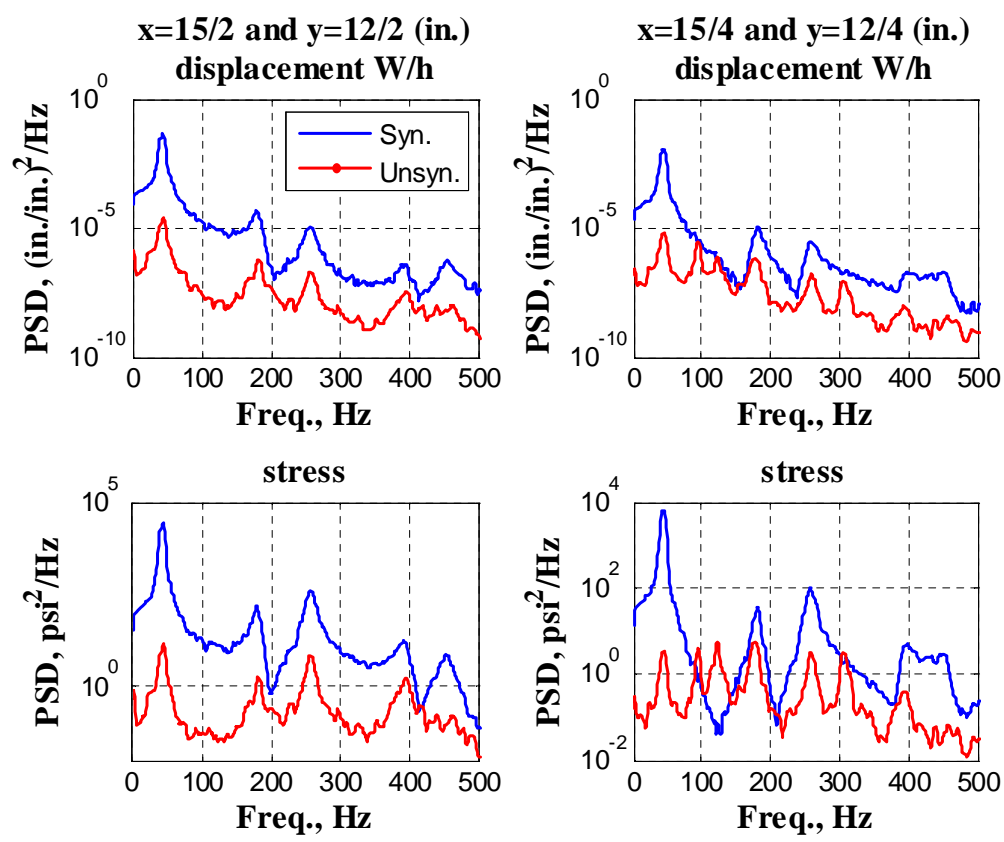

Figure 2. Displacement and stress PSD for synchronized and unsynchronized loads at $x=15 / 2$ in. and $y=12 / 2$ in. and $x=15 / 4$ in. and $y=12 / 4$ in 


\subsection{Panel Fatigue Life under Synchronized and Unsynchronized Loads.}

The MCS technique is adopted for the accurate prediction of fatigue life of panels under random synchronized and unsynchronized processes. The MCS consists of taking a number of various samples of load pressure distribution and evaluate the RMS maximum stress response of each sample at a time. With the results from many samples, a statistical distribution is obtained and used to find a mean value of the RMS responses. Fatigue life results are based on 10 samples of 1 sec. each where the first 0.125 sec. has been discarded to eliminate the initial transient response. The MCS technique has been proven to be effective for synchronized loadings where a small number of samples (based on one random ISEED number for each sample and all finite elemnts) yield pretty quickly to a converged fatigue estimate. However, the convergence for the unsynchronized loading case (each of the $32 \times 32=1024$ elements is excited by a different ISEED number) has shown to be extremely slow and computationally costly. This issue again makes the unsynchronized problem suitable for PCM where the simulated random load (different ISEED) could be distributed among different nodes (processors) instead of just one.

Table 5. Fatigue life prediction for simply-supported panel (10 samples)

\begin{tabular}{|l|c|c|}
\hline SPL $=100$ dB & Synchronized & Unsynchronized \\
\hline Fatigue Life Prediction (years) & 85.47 & $8.97 \times 10^{7}$ (Infinity) \\
\hline
\end{tabular}

Fatigue results shown in Table 5 are based on the maximum bending stress with S-N properties of $\beta=4.8$ and $K=1.52 \times 10^{25}$. These fatigue life prediction results
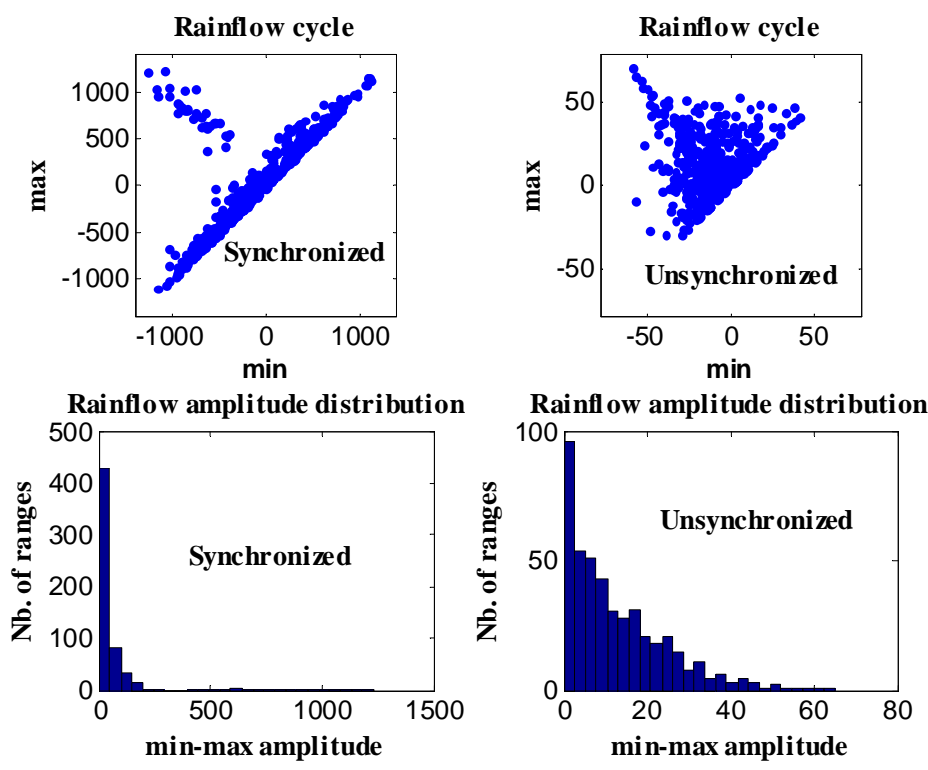

Figure 3. Rainflow cycle and rainflow amplitude distributions for synchronized and unsynchronized loads 
suggest the following two questions: 1) is the traditional synchronized random analysis too conservative for structures subjected to highly unsynchronized loads, and 2) if the SPL is high enough to require a nonlinear analysis for synchronized loads, would it be valid just to consider the linear analysis for highly unsynchronized loads at high SPL since their responses might be within the linear range? Certainly, much more investigations are needed for the unsynchronized loading cases at higher SPL including the consideration of nonlinear large deflection effects.

For the synchronized load, the maximum stress is at the panel center, whereas for the unsynchronized the maximum stress alternated between the center node (No. 481 ) and its eight adjacent nodes $(449,450,451,480,482,511,512,513)$. Figure 3 gives more detailed information about the characteristics of the stress responses yielding to the fatigue results in Table 5. The differences shown in both figures are an indication on how the stress response differs as a result of synchronized versus unsynchronized loading. This means that even if both responses were obtained from the same $\mathrm{SPL}=100 \mathrm{~dB}$, their fatigue characteristics are quite different due to the different stress min-max/rainflow amplitude distributions. For instance, the horizontal coordinate axis in the min-max amplitudes figures clearly indicates that the min-max amplitudes for the unsynchronized case are about a fourth of the min-max amplitudes for the synchronized loading case. Consequently, higher fatigue life prediction is expected for the unsynchronized case as shown in Table 5.

\subsection{Unsynchronized Response of Panel with Clamped-Simply Supported Boundary Conditions.}

A clamped-simply supported (C-SS-SS-C) plate clamped on edges $\mathrm{x}=0$ and $\mathrm{y}=0$, and simply supported on edges $\mathrm{x}=15$ in. and $\mathrm{y}=12$ in. $(\mathrm{x}=38.1 \mathrm{~cm} \& \mathrm{y}=30.48 \mathrm{~cm})$, with similar geometrical and material properties as in the previous section, is studied next. Figure 4 shows the first twenty-two unsymmetrical natural modes normalized with respect to the thickness, $\mathrm{h}=0.04$ in. $(0.1016 \mathrm{~cm})$, with their corresponding frequencies using a $24 \times 20$ mesh (480 elements). In order to reduce the computational time, results in this section are obtained from a mesh size of $24 \times 20$, which is adequate for 20 modes.

For the plate with mixed boundary conditions under synchronized or unsynchronized load for which no closed-form solution is readily available or exists, the most suitable approach is the MCS. For both loading conditions, the location of maximum displacement is situated approximately 8.75 inches $(22.225 \mathrm{~cm})$ away from the short clamped edge and 5.4 inches $(13.716 \mathrm{~cm}$ ) away from the long clamped edge (element 278 or node 243$)$.

The MCS for modal and mesh convergence of the unsynchronized loading case is numerically tedious and computationally costly, since each plate element is excited by a different random load or ISEED number at each of the ten-sample runs. This means that 480 pressure time histories with each time history containing $2^{16}$ points must be generated prior to the numerical integration. For the numerical simulation a 

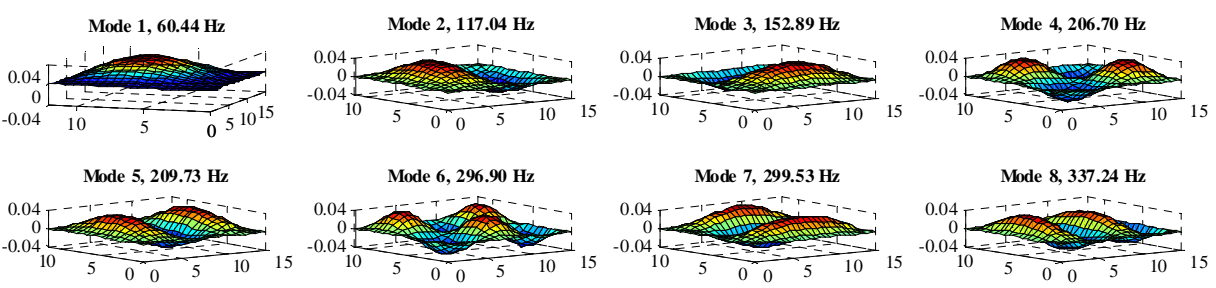

Mode 9, 352.21 Hz
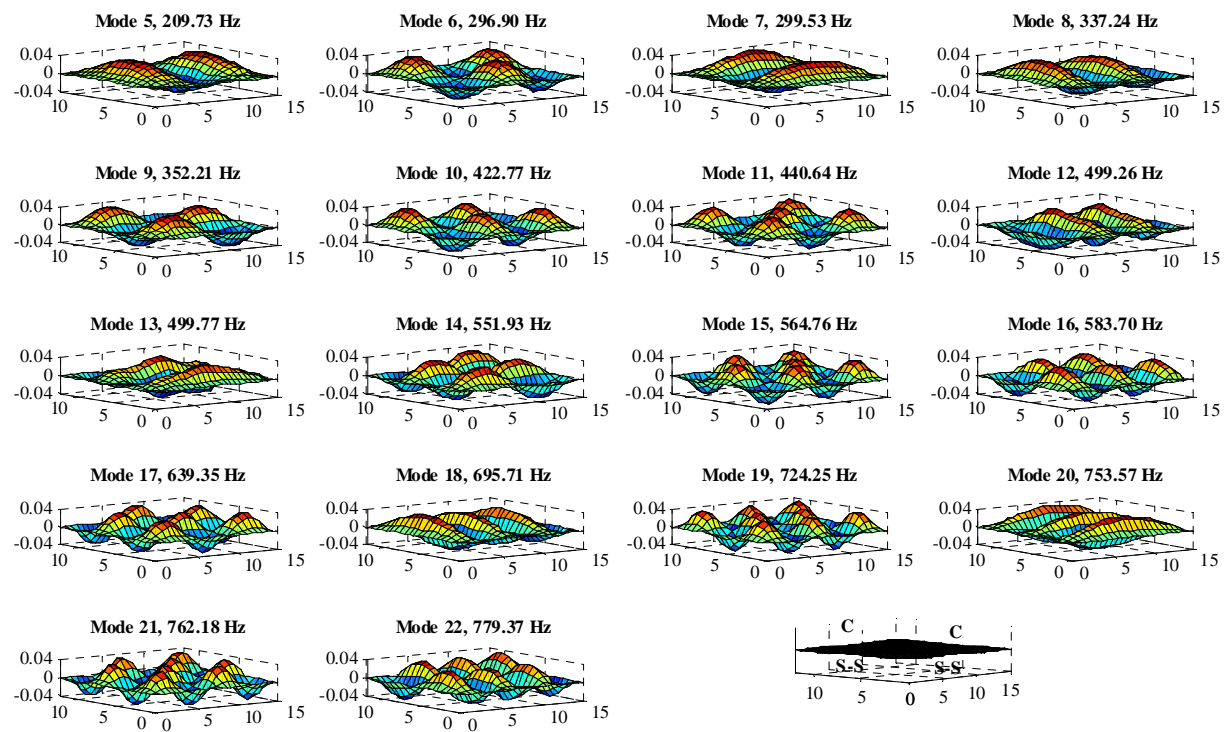

Figure 4. C-SS-SS-C panel modes and frequencies

sample run for unsynchronized loading on a $24 \times 20$ mesh with twenty-two modes and $\Delta t=1 / 2^{16}$ sec. took approximately 12 hours. The maximum stress is located along the long clamped edge at the node perpendicular to the maximum displacement. For the $24 \times 20$ mesh, this corresponds to element 14 or node 13 . The stress for the C-SSSS-C panel was found to be very sensitive to the location of the maximum curvature $\{\kappa\}$ which varies considerably with the mesh size.

Figure 5 presents the PSD of the maximum displacement (element 278 or node 243), and at the maximum stress (element 14 or node 13) for both synchronized and unsynchronized loading cases. The synchronized response results were generated using ISEED $=12357$ yielding to an RMS $\left(\mathrm{W}_{\max } / \mathrm{h}\right)$ of 0.410 , and RMS stress value of $477.49 \mathrm{psi}(3.292 \mathrm{MPa})$.

A first trial analysis of displacement PSD indicated that 6 asymmetric modes for the synchronized and 8 asymmetric modes for the unsynchronized loading cases were required for converged displacement responses. A refined analysis showed that more asymmetric modes contributed to both responses but that they did not appear in the PSD because their mode shapes were close to zero at that location (node 243). A clear example is given by mode $2(117.04 \mathrm{~Hz})$ 

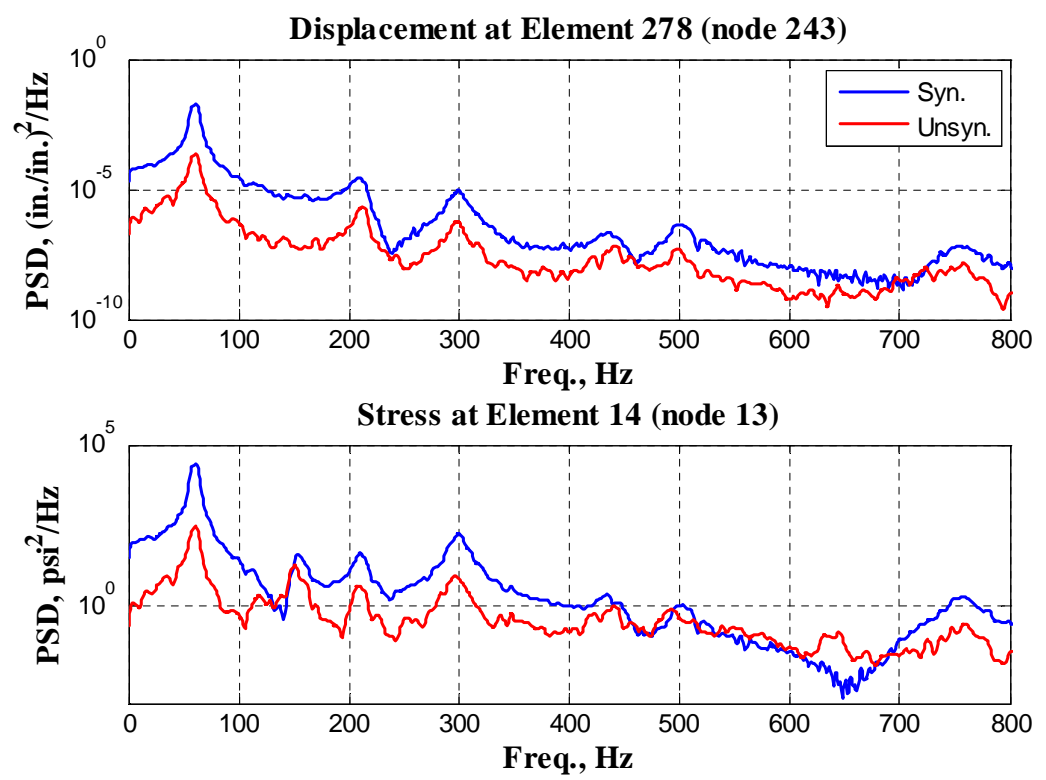

Figure 5. Maximum displacement and stress PSD for synchronized and unsynchronized loads.

A first trial analysis of displacement PSD indicated that 6 asymmetric modes for the synchronized and 8 asymmetric modes for the unsynchronized loading cases were required for converged displacement responses. A refined analysis showed that more asymmetric modes contributed to both responses but that they did not appear in the PSD because their mode shapes were close to zero at that location (node 243). A clear example is given by mode $2(117.04 \mathrm{~Hz})$ in Fig. 4, where the figure reveals that at node 243 its modal contribution is very small or zero. It is observed for the stress PSD that more modes (peaks) appear for the unsynchronized than for the synchronized loading.

Table 6. Fatigue life prediction for C-SS-SS-C panel (10 samples)

\begin{tabular}{|l|c|c|}
\hline SPL $=100 \mathrm{~dB}$ & Synchronized & Unsynchronized \\
\hline Fatigue Life Prediction (years) & 77.42 (Infinity) & $10.55 \times 10^{5}$ (Infinity) \\
\hline
\end{tabular}

Fatigue lives shown in Table 6 are predicted at node 13 where the stress is the highest and most critical. Results are based on the mean value of the fatigue life of ten samples.

\section{Conclusion}

The present work has studied the RMS responses of a simply supported and a clamped-simply supported panel subjected to synchronized and unsynchronized loading, respectively. Classic analytical solutions of the random responses are generally 
confined to simple boundary conditions where the mode shape solutions are known. Even in those simple cases, response solutions have neglected the asymmetric modes for the synchronized case due to mathematical limitations. The present work has shown for the first time that panels under unsynchronized loads excite both symmetric and asymmetric modes, and that the unsynchronized load characteristics lower the RMS values and increase the fatigue life predictions.

These conclusions appear to be quite intuitive but they are a first attempt to explore and understand the real loading conditions. It is likely that real random pressure excitation characteristics are neither fully spatially correlated nor uncorrelated. In such case developing a procedure applicable for analysis with an arbitrary spatial input correlation is deemed an important part of future work. In addition, in order to verify these conclusions, more simulation studies and further experimental data of structures subjected to uniform random pressure loads that are unsynchronized in time are needed.

The computational burden encountered in solving the unsynchronized loading case required the necessity of considering Parallel Computing Methods (PCM). Parallel computing methods greatly reduce the computational time by splitting the load generation into multiple processors (nodes).

\section{REFERENCES}

1. Arnold, R. R. and VAicaitis, R. R.: Nonlinear Response and Fatigue of Surface Panels by the Time Domain Monte Carlo Approach, WRDC-TR-90-3081, Wright Laboratory, WPAFB, OH, May 1992.

2. Mei, C. and Wolfe, H. F.: On large deflection analysis in acoustic design, in Random Vibrations - Status and Recent Developments. The Stephen H. Crandall Festschrift, Editors: I. Elishakoff and R. H. Lyon, Elsevier Applied Science Publishers, Amsterdam 1986, 279-302.

3. Mei, C., Dhainaut, J. M., Duan, B., Spottswood, S. M. and Wolfe, H. F.: Nonlinear Random Response of Composite Panels in an Elevated Thermal Environment, $A F R L-V A-W P-T R$-2000-3049, Air Force Research Laboratory, WPAFB, OH, October 2000.

4. McEvan, M. J., Wright, J .R., Copper, J .E. and Leung, A.Y.T.: A finite element/modal technique for nonlinear plate and stiffened panel response prediction. AIAA 2001-1595, $42^{\text {nd }}$ Structures, Structural Dynamics and Materials Conference, Seattle, WA, April 2001 (CD-ROM).

5. Rizzi, S. A. and Muravyov, A. A.: Improved equivalent linearization implementations using nonlinear stiffness evaluation. NASA TM-2001-210838, March 2001.

6. Muravyov, A. A. and Rizzi, S. A.: Determination of nonlinear stiffness with application to random vibration of geometrically nonlinear structures. Computers and Structures, 81(15), (2003), 1513-1523.

7. Dhainaut, J. M. and Mei, C.: Nonlinear response and fatigue life of isotropic panels subjected to nonwhite pressure fluctuations. AIAA 2002-1635, $43^{\text {rd }}$ Structures, Structural Dynamics, and Materials Conference, Denver, Colorado, April, 2002, 22-25. 
8. Przekop, A., Guo, X., Azzouz, S. and Mei, C.: Reinvestigation of nonlinear random response of shallow shells using finite element modal formulation. AIAA 2004-1553, $45^{\text {th }}$ Structures, Structural Dynamics and Materials Conference, Palm Spring, CA, April 2004 (CD-ROM).

9. Mei, C. and Wentz, K. R.: Analytical and experimental nonlinear response of rectangular panels to acoustic excitation. AIAA 82-0733, $23^{\text {rd }}$ Structures, Structural Dynamics and Materials Conference, New Orleans, LA, May 1982, 514-520.

10. Hollkamp, J. J., Gordon, R. W. and Spottswood, S. M.: Nonlinear sonic fatigue response prediction from finite element modal models: A comparison with experiments. AIAA 2003-1709, 44 ${ }^{\text {th }}$ Structures, Structural Dynamics and Materials Conference, Norfolk, VA, April 2003 (CD-ROM).

11. Bogner, F. K., Fox, R. L. and Schmidt, L. A.: The generation of inter-element compatible stiffness and mass matrices by the use of interpolation formulas. AFFDLTR-66-80, Wright-Patterson AFB, OH, 1996, 396-443.

12. BARLOW, J.: Optimal stress locations in finite element models. International Journal for Numerical Methods in Engineering, 10, (1976), 243-251.

13. Miner, M. A.: Cumulative damage in fatigue. Journal of Applied Mechanics, 12, (1945), 159-164.

14. Langley, R. S. and McWilliam, S.: Mathematical models for structural reliability analysis. F. Casciati and B. Roberts (eds), CRC Press, July 1996, 333-334.

15. Dowling, N. E.,: Fatigue failure predictions for complicated stress-strain histories. Journal of Materials, 7, (1972), 71-87.

16. WAFO - A Matlab Toolbox for Analysis of Random Waves and Loads, Version 2.0.02, Lund Institute of Technology, Lund University, 2000.

17. Bishop, N. W. N. and Sherratt, F.: A theoretical solution for the estimation of rainflow ranges from power spectral density data. Fatigue Fracture Engineering Material Structures, 13(4), (1990), 311-326. 